\title{
Generalized Cattaneo-Maxwell diffusion equation with fractional derivatives. Dispersion relations
}

\author{
Kostrobij P. ${ }^{1}$, Markovych B. ${ }^{1}$, Viznovych O. ${ }^{1}$, Zelinska I. ${ }^{1}$, Tokarchuk M. ${ }^{1,2}$ \\ ${ }^{1}$ Lviv Polytechnic National University, \\ 12 S. Bandera Str., 79013, Lviv, Ukraine \\ ${ }^{2}$ Institute for Condensed Matter Physics \\ of the National Academy of Sciences of Ukraine, \\ 1 Svientsitskii Str., 79011, Lviv, Ukraine
}

(Received 1 March 2019; Revised 23 May 2019; Accepted 30 May 2019)

\begin{abstract}
The new non-Markovian diffusion equations of ions in spatially heterogeneous environment with fractal structure and generalized Cattaneo-Maxwell diffusion equation with taking into account the space-time nonlocality are obtained. Dispersion relations for the Cattaneo-Maxwell-type diffusion equation with taking into account the space-time nonlocality in fractional derivatives are found. The frequency spectrum, phase and group velocities are calculated. It is shown that it has a wave behavior with discontinuities, which are also manifested in behavior of the phase velocity.

Keywords: generalized diffusion equation, nonequilibrium statistical operator, Renyi statistics, multifractal time, spatial fractality, nonlocality of space-time.
\end{abstract}

2000 MSC: $82 \mathrm{C} 05,82 \mathrm{C} 70$

UDC: 538.93

DOI: $10.23939 / \mathrm{mmc} 2019.01 .058$

\section{Introduction}

Fractional integrals and derivatives [1-5] are actively used in researches of anomalous diffusion in porous media [5-16], disordered systems [17-30], plasma physics [31-36], turbulent [37-39], kinetic and reaction-diffusion processes [39-48], biological systems [49-51], etc. [5, 52,53]. An actual problem for description of nonequilibrium processes in complex systems is construction of generalized diffusion and wave equations $[54,55]$ using fractional integrals and derivatives. The dispersion of heat waves in a dissipative environment using the Cattaneo-Maxwell heat diffusion equation with fractional derivatives has breen investigated in Ref. [56]. On the basis of this equation, the frequency spectrum, phase and group velocities of propagation of heat waves in a dissipative environment have been investigated.

In Refs. [30,57-60], the statistical approach has been developed for obtaining the space-time nonlocal transport equations with fractional derivatives using the Zubarev nonequilibrium statistical operator method [61-64] and the Liouville equation with fractional derivatives, proposed by Tarasov [40].

In the second section, based on the statistical approach within the Renyi statistics, we have obtained a generalized diffusion equation with fractional derivatives for the nonequilibrium average value of the number density of particles. This equation is nonlocal in space and time. In the third section, within the Gibbs statistics and approximation of constant diffusion coefficient, the frequency spectrum of the Cattaneo-Maxwell-type diffusion equation for the nonequilibrium average value of the number density of particles has been obtained. The frequency spectrum, phase and group velocities have been calculated, depending on order of the fractional derivative, characteristic relaxation time and value of the diffusion coefficient.

\section{Generalized diffusion equations with fractional derivatives}

To describe the diffusion processes of particle in heterogeneous environments with fractal structure, one of main parameters of the reduced description is the nonequilibrium density of particle numbers 
$n(\boldsymbol{r} ; t)=\langle\hat{n}(\boldsymbol{r})\rangle_{\alpha}^{t}$, where $\hat{n}(\boldsymbol{r})=\sum_{j=1}^{N} \delta\left(\boldsymbol{r}-\boldsymbol{r}_{j}\right)$ is the microscopic density of the particle. The corresponding generalized diffusion equation for $n(\boldsymbol{r} ; t)$ can be obtained on the base of approach [57], by using the Zubarev nonequilibrium statistical operator method within the Renyi statistics for solution of the Liouville equations with fractional derivatives,

$$
\begin{gathered}
\frac{\partial}{\partial t}\langle\hat{n}(\boldsymbol{r})\rangle_{\alpha}^{t}=D_{r}^{\alpha} \cdot \int d \mu_{\alpha}\left(\boldsymbol{r}^{\prime}\right) \int_{-\infty}^{t} e^{\varepsilon\left(t^{\prime}-t\right)} D_{q}\left(\boldsymbol{r}, \boldsymbol{r}^{\prime} ; t, t^{\prime}\right) \cdot D_{r^{\prime}}^{\alpha} \beta \nu^{*}\left(\boldsymbol{r}^{\prime} ; t^{\prime}\right) d t^{\prime}, \\
D_{q}\left(\boldsymbol{r}, \boldsymbol{r}^{\prime} ; t, t^{\prime}\right)=\left\langle\hat{\boldsymbol{v}}(\boldsymbol{r}) T\left(t, t^{\prime}\right) \hat{\boldsymbol{v}}\left(\boldsymbol{r}^{\prime}\right)\right\rangle_{\alpha, r e l}^{t}
\end{gathered}
$$

is the generalized coefficient of particle diffusion within the Renyi statistics, averaging of which is performed with a power-law Renyi distribution,

$$
\rho_{\text {rel }}(t)=\frac{1}{Z_{R}(t)}\left(1-\frac{q-1}{q} \beta\left(H-\int d \mu_{\alpha}(\boldsymbol{r}) \nu^{*}(\boldsymbol{r} ; t) \hat{n}(\boldsymbol{r})\right)\right)^{\frac{1}{q-1}},
$$

where

$$
Z_{R}(t)=\hat{I}^{\alpha}(1, \ldots, N) \hat{T}(1, \ldots, N)\left(1-\frac{q-1}{q} \beta\left(H-\int d \mu_{\alpha}(\boldsymbol{r}) \nu^{*}(\boldsymbol{r} ; t) \hat{n}(\boldsymbol{r})\right)\right)^{\frac{1}{q-1}}
$$

is the partition function of the relevant distribution function, $H$ is a Hamiltonian of system, $0<q \leqslant 1$, $q$ is the Renyi parameter;

$$
\nu^{*}(\boldsymbol{r} ; t)=\frac{\nu(\boldsymbol{r} ; t)}{1+\frac{q-1}{q} \int d \mu_{\alpha}(\boldsymbol{r}) \nu(\boldsymbol{r} ; t)\langle\hat{n}(\boldsymbol{r})\rangle_{\alpha}^{t}},
$$

$T\left(t, t^{\prime}\right)=\exp _{+}\left(-\int_{t^{\prime}}^{t}\left(1-P_{\text {rel }}\left(t^{\prime \prime}\right)\right) i L_{\alpha} d t^{\prime \prime}\right)$ is the evolution operator in time containing the projection; $\exp _{+}$is the ordered exponentia, $P_{r e l}\left(t^{\prime \prime}\right)$ is the generalized Kawasaki-Gunton projection operator depended on a structure of the relevant statistical operator (distribution function), $\rho_{\text {rel }}\left(x^{N} ; t^{\prime}\right) . i L_{\alpha}$ is the Liouville operator for a system of particles in heterogeneous environment with fractal structure. Parameter $\nu(\boldsymbol{r} ; t)$ is the chemical potential of particle, which is determined from the self-consistency condition,

$$
\langle\hat{n}(\boldsymbol{r})\rangle_{\alpha}^{t}=\langle\hat{n}(\boldsymbol{r})\rangle_{\alpha, r e l}^{t} .
$$

$\beta=1 / k_{\mathrm{B}} T, k_{\mathrm{B}}$ is the Boltzmann constant, $T$ is the equilibrium value of temperature; $\hat{\boldsymbol{v}}(\boldsymbol{r})=$ $\sum_{j=1}^{N} \boldsymbol{v}_{j} \delta\left(\boldsymbol{r}-\boldsymbol{r}_{j}\right)$ is microscopic flux density of the particle. The average values in Eq. (2) are calculated by (see Ref. [57])

$$
\langle(\ldots)\rangle_{\alpha, r e l}^{t}=\hat{I}^{\alpha}(1, \ldots, N) \hat{T}(1, \ldots, N)(\ldots) \rho_{r e l}\left(x^{N} ; t\right),
$$

where $\hat{I}^{\alpha}(1, \ldots, N)$ for system of $N$ particles has the form

$$
\hat{I}^{\alpha}(1, \ldots, N)=\hat{I}^{\alpha}(1) \ldots \hat{I}^{\alpha}(N), \quad \hat{I}^{\alpha}(j)=\hat{I}^{\alpha}\left(\boldsymbol{r}_{j}\right) \hat{I}^{\alpha}\left(\boldsymbol{p}_{j}\right)
$$

and defines the integration operation,

$$
\hat{I}^{\alpha}(x) f(x)=\int_{-\infty}^{\infty} f(x) d \mu_{\alpha}(x), \quad d \mu_{\alpha}(x)=\frac{|x|^{\alpha}}{\Gamma(\alpha)} d x .
$$

The operator $\hat{T}(1, \ldots, N)=\hat{T}(1) \ldots \hat{T}(N)$ defines the operation

$$
\hat{T}\left(x_{j}\right) f\left(x_{j}\right)=\frac{1}{2}\left(f\left(\ldots, x_{j}^{\prime}-x_{j}, \ldots\right)+f\left(\ldots, x_{j}^{\prime}+x_{j}, \ldots\right)\right) .
$$

In the generalized diffusion equation $(1), d^{\alpha}$ is a fractional differential [65] that is defined by

$$
d^{\alpha} f(x)=\sum_{j=1}^{2 N} D_{x_{j}}^{\alpha} f(x)\left(d x_{j}\right)^{\alpha}
$$


where

$$
D_{x}^{\alpha} f(x)=\frac{1}{\Gamma(n-\alpha)} \int_{0}^{x} \frac{f^{(n)}(z)}{(x-z)^{\alpha+1-n}} d z
$$

is the Caputo fractional derivative $[1,2,66,67], n-1<\alpha<n, f^{(n)}(z)=\frac{d^{n}}{d z^{n}} f(z)$ with the properties $D_{x_{j}}^{\alpha} 1=0$ and $D_{x_{j}}^{\alpha} x_{l}=0,(j \neq l)$.

At $q=1$, the generalized diffusion equation within the Renyi statistics goes into the generalized diffusion equation within the Gibbs statistics with fractional derivatives. If $q=1$ and $\alpha=1$, we obtain the generalized diffusion equation within the Gibbs statistics. In the Markov approximation, the generalized coefficient of mutual diffusion in time and space has the form $D_{q}\left(\boldsymbol{r}, \boldsymbol{r}^{\prime} ; t, t^{\prime}\right) \approx D_{q} \delta(t-$ $\left.t^{\prime}\right) \delta\left(\boldsymbol{r}-\boldsymbol{r}^{\prime}\right)$, by excluding the parameter $\nu^{*}\left(\boldsymbol{r}^{\prime} ; t^{\prime}\right)$ via the self-consistency condition, we obtain the diffusion equation with fractional derivatives from Eq. (1)

$$
\frac{\partial}{\partial t}\langle\hat{n}(\boldsymbol{r})\rangle_{\alpha}^{t}=D_{q} D_{r}^{2 \alpha} \nu^{*}(\boldsymbol{r} ; t)
$$

The generalized diffusion equation takes into account spatial fractality of system and memory effects in the generalized coefficient of particle diffusion $D_{q}\left(\boldsymbol{r}, \boldsymbol{r}^{\prime} ; t, t^{\prime}\right)$ within the Renyi statistics. Obviously, spatial fractality of system influences on particle transport processes that may manifest as multifractal time with characteristic relaxation times. It is known that the nonequilibrium correlation functions $D_{q}\left(\boldsymbol{r}, \boldsymbol{r}^{\prime} ; t, t^{\prime}\right)$ can not be exactly calculated, therefore the some approximations based on physical reasons are used. In the time interval $-\infty \div t$, ion transport processes in spatially heterogeneous system can be characterized by a set of relaxation times that are associated with the nature of interaction between of the particle and particles of environment with fractal structure. For opening of multifractal time in the generalized diffusion equation, we use the following approach for the generalized coefficient of the particle diffusion

$$
D_{q}\left(\boldsymbol{r}, \boldsymbol{r}^{\prime} ; t, t^{\prime}\right)=W\left(t, t^{\prime}\right) \bar{D}_{q}\left(\boldsymbol{r}, \boldsymbol{r}^{\prime}\right),
$$

where $W\left(t, t^{\prime}\right)$ can be defined as the time memory function. In view of this, Eq. (1) can be represented as

$$
\frac{\partial}{\partial t}\langle\hat{n}(\boldsymbol{r})\rangle_{\alpha}^{t}=\int_{-\infty}^{t} e^{\varepsilon\left(t^{\prime}-t\right)} W\left(t, t^{\prime}\right) \Psi\left(\boldsymbol{r} ; t^{\prime}\right) d t^{\prime},
$$

where

$$
\Psi\left(\boldsymbol{r} ; t^{\prime}\right)=\int d \mu_{\alpha}\left(\boldsymbol{r}^{\prime}\right) D_{r}^{\alpha} \cdot \bar{D}_{q}\left(\boldsymbol{r}, \boldsymbol{r}^{\prime}\right) \cdot D_{r^{\prime}}^{\alpha} \beta \nu^{*}\left(\boldsymbol{r}^{\prime} ; t^{\prime}\right) .
$$

Further we apply the Fourier transform to Eq. (10), and as a result we get in frequency representation

$$
i \omega n(\boldsymbol{r} ; \omega)=W(\omega) \Psi(\boldsymbol{r} ; \omega) .
$$

We can represent frequency dependence of the memory function in the following form

$$
W(\omega)=\frac{(i \omega)^{1-\xi}}{1+(i \omega \tau)^{\xi}}, \quad 0<\xi \leqslant 1,
$$

where the introduced relaxation time $\tau$ characterizes of the particle transport processes in system. Then Eq. (12) can be represented as

$$
\left(1+(i \omega \tau)^{\xi}\right) i \omega n(\boldsymbol{r} ; \omega)=(i \omega)^{1-\xi} \Psi(\boldsymbol{r} ; \omega) .
$$

Further we use the Fourier transform to fractional derivatives of functions,

$$
L\left({ }_{0} \mathrm{D}_{t}^{1-\xi} f(t) ; i \omega\right)=(i \omega)^{1-\xi} L(f(t) ; i \omega),
$$

where ${ }_{0} \mathrm{D}_{t}^{1-\xi} f(t)=\frac{1}{\Gamma(\xi)} \frac{d}{d t} \int_{0}^{t} \frac{f(\tau)}{(t-\tau)^{1-\xi}} d \tau$ is the Riemann-Liouville fractional derivative. By using it, the inverse transformation of Eq. (14) to time representation gives the Cattaneo-type generalized diffusion equation with taking into account spatial fractality, in the expanded form 


$$
{ }_{0} \mathrm{D}_{t}^{2 \xi} n(\boldsymbol{r} ; t) \tau^{\xi}+{ }_{0} \mathrm{D}_{t}^{\xi} n(\boldsymbol{r} ; t)=\int d \mu_{\alpha}\left(\boldsymbol{r}^{\prime}\right) D_{r}^{\alpha} \cdot \bar{D}_{q}\left(\boldsymbol{r}, \boldsymbol{r}^{\prime}\right) \cdot D_{r^{\prime}}^{\alpha} \beta \nu^{*}\left(\boldsymbol{r}^{\prime} ; t\right)
$$

is the new Cattaneo-type generalized equation within the Renyi statistics with time and spatial nonlocality. At $q=1$ from Eq. (16), we get the Cattaneo-type generalized equation within the Gibbs statistics with time and spatial nonlocality.

Eq. (16) contains significant spatial heterogeneity in $\bar{D}_{q}\left(\boldsymbol{r}, \boldsymbol{r}^{\prime}\right)$. If we neglect spatial heterogeneity,

$$
\bar{D}_{q}\left(\boldsymbol{r}, \boldsymbol{r}^{\prime}\right)=\bar{D}_{q} \delta\left(\boldsymbol{r}-\boldsymbol{r}^{\prime}\right),
$$

we get the Cattaneo-type diffusion equation with of space-time nonlocality and constant coefficients of diffusion within the Renyi statistics

$$
{ }_{0} \mathrm{D}_{t}^{2 \xi} n(\boldsymbol{r} ; t) \tau^{\xi}+{ }_{0} \mathrm{D}_{t}^{\xi} n(\boldsymbol{r} ; t)=\bar{D}_{q} D_{r}^{2 \alpha} \beta \nu^{*}\left(\boldsymbol{r}^{\prime} ; t\right),
$$

or in the expanded form

$$
{ }_{0} \mathrm{D}_{t}^{2 \xi} n(\boldsymbol{r} ; t) \tau^{\xi}+{ }_{0} \mathrm{D}_{t}^{\xi} n(\boldsymbol{r} ; t)=\bar{D}_{q} D_{r}^{2 \alpha} \beta \frac{\nu(\boldsymbol{r} ; t)}{1+\frac{q-1}{q} \int d \mu_{\alpha}(\boldsymbol{r}) \nu(\boldsymbol{r} ; t)\langle\hat{n}(\boldsymbol{r})\rangle_{\alpha}^{t}},
$$

and at $q=1$ we get the Cattaneo-type diffusion equation with of space-time nonlocality and constant coefficients of diffusion within the Gibbs statistics,

$$
{ }_{0} \mathrm{D}_{t}^{2 \xi} n(\boldsymbol{r} ; t) \tau^{\xi}+{ }_{0} \mathrm{D}_{t}^{\xi} n(\boldsymbol{r} ; t)=\bar{D} D_{r}^{2 \alpha} \beta \nu(\boldsymbol{r} ; t) .
$$

\section{Dispersion relations for the time-space-fractional Cattaneo-Maxwell diffusion equation}

Using the self-consistent condition (5) and the approved approximations, Eq. (20) can be written as

$$
{ }_{0} \mathrm{D}_{t}^{2 \xi} n(\boldsymbol{r} ; t) \tau^{\xi}+{ }_{0} \mathrm{D}_{t}^{\xi} n(\boldsymbol{r} ; t)-\overline{D^{\prime}} D_{r}^{2 \alpha} n(\boldsymbol{r} ; t)=0,
$$

where $\overline{D^{\prime}}$ is the renormalized diffusion coefficient. For simplicity, we consider the one-dimensional case and a solution of Eq. (21) will be sought in the form of the plane wave,

$$
n(x ; t) \sim e^{-i \omega t+i k x},
$$

then we get the corresponding frequency spectrum,

$$
\left(\tau^{\xi}(-i \omega)^{2 \xi}+(-i \omega)^{\xi}\right)-\overline{D^{\prime}}(i k)^{2 \alpha}=0 .
$$

It should be noted that the real part $\omega_{r}(k)$ of the frequency spectrum $\omega(k)=\omega_{r}(k)+i \omega_{i}(k)$ describes propagation of process, the imaginary part of $\omega_{i}(k)$ describes damping of process in time. The real part $k(\omega)_{r}$ of the wave spectrum $k(\omega)=k(\omega)_{r}+i k(\omega)_{i}$ describes propagation of the process, the imaginary part $k(\omega)_{i}$ describes damping of the process in space. In addition, the real part of the frequency spectrum determines the wave dependence of the phase and group velocities of process:

$$
v_{p}(k)=\frac{1}{k} \omega_{r}(k), \quad v_{g}(k)=\frac{\partial}{\partial k} \omega_{r}(k),
$$

and the real part of the wave spectrum determines the frequency dependence of the phase velocity of process,

$$
v_{p}(\omega)=\frac{\omega}{k(\omega)_{r}} .
$$

Mathematical Modeling and Computing, Vol. 6, No. 1, pp. 58-68 (2019) 
First, let us consider the case $\alpha=1$ and $0<\xi<1$. The similar problem is solved in Ref. [56] at investigating dispersion relations for the Cattaneo-Maxwell heat transfer equation with fractional derivatives. At $\alpha=1$, we get the equation

$$
\left(\tau^{\xi}(-i \omega)^{2 \xi}+(-i \omega)^{\xi}\right)+\overline{D^{\prime}} k^{2}=0
$$

the solution of which has the form:

$$
(-i \omega)^{\xi}=\frac{-1 \pm \sqrt{1-4 \tau^{\xi} \overline{D^{\prime}} k^{2}}}{2 \tau^{\xi}}
$$

or

$$
\omega^{\xi}=i^{\xi} \frac{-1 \pm \sqrt{1-4 \tau^{\xi} \overline{D^{\prime}} k^{2}}}{2 \tau^{\xi}}
$$

Next we find imaginary and real parts of the frequency by putting $\omega=|\omega| e^{i \theta},|\omega|>0,-\pi<\theta<\pi$, then we get

$$
|\omega|^{\xi} e^{i \xi \theta}=e^{i \xi \frac{\pi}{2}} \frac{-1 \pm \sqrt{1-4 \tau^{\xi} \overline{D^{\prime}} k^{2}}}{2 \tau^{\xi}} .
$$

The solution of Eq. (27) will be rewritten depending on a sign of expression under the root, $B(k)=$ $1-4 \tau^{\xi} \overline{D^{\prime}} k^{2}$.

In the case of $B(k) \geqslant 0$ we have that

$$
|\omega|=\frac{1}{\tau}\left|\frac{-1 \pm \sqrt{1-4 \tau^{\xi} \overline{D^{\prime}} k^{2}}}{2}\right|^{\frac{1}{\xi}}, \quad \theta=\frac{\pi}{2}+\frac{\pi}{\xi} .
$$

Then the real and imaginary parts of the frequency spectrum $\omega(k)=\omega_{r}(k)+i \omega_{i}(k)$ will have the form:

$$
\begin{aligned}
& \omega_{r}(k)=|\omega| \cos \theta=-\frac{1}{\tau}\left|\frac{-1 \pm \sqrt{1-4 \tau^{\xi} \overline{D^{\prime}} k^{2}}}{2}\right|^{\frac{1}{\xi}} \sin \frac{\pi}{\xi}, \quad 0 \leqslant k \leqslant \frac{1}{\sqrt{4 \tau^{\xi} \overline{D^{\prime}} k^{2}}} \\
& \omega_{i}(k)=|\omega| \sin \theta=\frac{1}{\tau}\left|\frac{-1 \pm \sqrt{1-4 \tau^{\xi} \overline{D^{\prime}} k^{2}}}{2}\right|^{\frac{1}{\xi}} \cos \frac{\pi}{\xi}, \quad 0 \leqslant k \leqslant \frac{1}{\sqrt{4 \tau^{\xi} \overline{D^{\prime}} k^{2}}} .
\end{aligned}
$$

According to definitions of the phases and group velocities:

$$
v_{p}(k)=\frac{\omega_{r}(k)}{k}, \quad v_{g}(k)=\frac{\partial}{\partial k} \omega_{r}(k),
$$

we obtain the following expressions for $v_{p}(k)$ and $v_{g}(k)$ :

$$
\begin{gathered}
v_{p}(k)=-\frac{1}{\tau k}\left|\frac{-1 \pm \sqrt{1-4 \tau^{\xi} \overline{D^{\prime}} k^{2}}}{2}\right|^{\frac{1}{\xi}} \sin \frac{\pi}{\xi}, \quad 0 \leqslant k \leqslant \frac{1}{\sqrt{4 \tau^{\xi} \overline{D^{\prime}} k^{2}}}, \\
v_{g}(k)=\mp \frac{2}{\tau \xi} \frac{\tau^{\xi} \overline{D^{\prime}} k^{2}}{\sqrt{1-4 \tau^{\xi} \overline{D^{\prime}} k^{2}}}\left(\frac{1 \pm \sqrt{1-4 \tau^{\xi} \overline{D^{\prime}} k^{2}}}{2}\right)^{\frac{1-\xi}{\xi}}, \quad 0 \leqslant k \leqslant \frac{1}{\sqrt{4 \tau^{\xi} \overline{D^{\prime}} k^{2}}} .
\end{gathered}
$$

In the case of $B(k)<0$ we have that

$$
|\omega|^{\xi} e^{i \xi \theta}=e^{i \xi \frac{\pi}{2}} \frac{-1 \pm i \sqrt{\left|1-4 \tau^{\xi} \overline{D^{\prime}} k^{2}\right|}}{2 \tau^{\xi}} .
$$


It is convenient to submit the fraction in an exponential form:

$$
\frac{-1 \pm i \sqrt{\left|1-4 \tau^{\xi} \overline{D^{\prime}} k^{2}\right|}}{2 \tau^{\xi}}=K e^{i \psi}
$$

where

$$
K=\left|\frac{-1 \pm i \sqrt{\left|1-4 \tau^{\xi} \overline{D^{\prime}} k^{2}\right|}}{2 \tau^{\xi}}\right|=\sqrt{\frac{\overline{D^{\prime}} k^{2}}{\tau^{\xi}}}, \quad \psi=\mp \arctan \sqrt{4 \tau^{\xi} \overline{D^{\prime}} k^{2}-1} .
$$

Taking this into account, we get that

$$
|\omega|^{\xi}=\sqrt{\frac{\overline{D^{\prime}} k^{2}}{\tau^{\xi}}}, \quad \xi \theta=\frac{\xi \pi}{2}+\psi
$$

or

$$
|\omega|=\left(\sqrt{\frac{\overline{D^{\prime}} k^{2}}{\tau^{\xi}}}\right)^{\frac{1}{\xi}}, \quad \theta=\frac{\pi}{2} \mp \frac{1}{\xi} \arctan \sqrt{4 \tau^{\xi} \overline{D^{\prime}} k^{2}-1} .
$$

Accordingly, for the real and imaginary parts of the frequency spectrum, we find that

$$
\begin{aligned}
& \omega_{r}(k)=|\omega| \cos \theta= \pm\left(\sqrt{\frac{\overline{D^{\prime}} k^{2}}{\tau^{\xi}}}\right)^{\frac{1}{\xi}} \sin \left(\frac{1}{\xi} \arctan \sqrt{4 \tau^{\xi} \overline{D^{\prime}} k^{2}-1}\right), \\
& \omega_{i}(k)=|\omega| \sin \theta=\left(\sqrt{\frac{\overline{D^{\prime}} k^{2}}{\tau^{\xi}}}\right)^{\frac{1}{\xi}} \cos \left(\frac{1}{\xi} \arctan \sqrt{4 \tau^{\xi} \overline{D^{\prime}} k^{2}-1}\right) .
\end{aligned}
$$

In this case, the phase and group velocities obtain the following form:

$$
\begin{aligned}
v_{p}(k)= & \pm \frac{1}{k}\left(\sqrt{\frac{\overline{D^{\prime}} k^{2}}{\tau^{\xi}}}\right)^{\frac{1}{\xi}} \sin \left(\frac{1}{\xi} \arctan \sqrt{4 \tau^{\xi} \overline{D^{\prime}} k^{2}-1}\right), \\
v_{g}(k)= & \pm \frac{1}{\xi k}\left(\sqrt{\frac{\overline{D^{\prime}} k^{2}}{\tau^{\xi}}}\right)^{\frac{1}{\xi}}\left(\sin \left(\frac{1}{\xi} \arctan \sqrt{4 \tau^{\xi} \overline{D^{\prime}} k^{2}-1}\right)\right. \\
& \left.+\frac{\cos \left(\frac{1}{\xi} \arctan \sqrt{4 \tau^{\xi} \overline{D^{\prime}} k^{2}-1}\right)}{\sqrt{4 \tau^{\xi} \overline{D^{\prime}} k^{2}-1}}\right) .
\end{aligned}
$$

Using the analytic expressions for the frequency spectrum, phase and group velocities in the cases $B(k) \geqslant 0$ and $B(k)<0$, numerical calculations are carried out at two values of the relaxation time $\tau=0.2,0.5$ and the diffusion coefficient $\overline{D^{\prime}}=0.5$ with different values of the order $\xi$ of the fractional derivative $\xi=0.3,0.5,0.8,1$. The results of the calculations are presented in Figs. $1-4$. For $\xi=1$, we have the usual Cattaneo-Maxwell equation with the corresponding behavior of the frequency spectrum. From analysis of the frequency spectrum for $\xi=0.3,0.5,0.8$, we see nonlinear wave behavior in both the propagation and the damping of the diffusion process. In the behavior of $\omega_{r}(k)$ there are discontinuities, which is also manifested in phase velocity, respectively. This may indicate a sharp change in the nature of the diffusion wave process. The group velocity has $\lambda$-like form with a sharp 

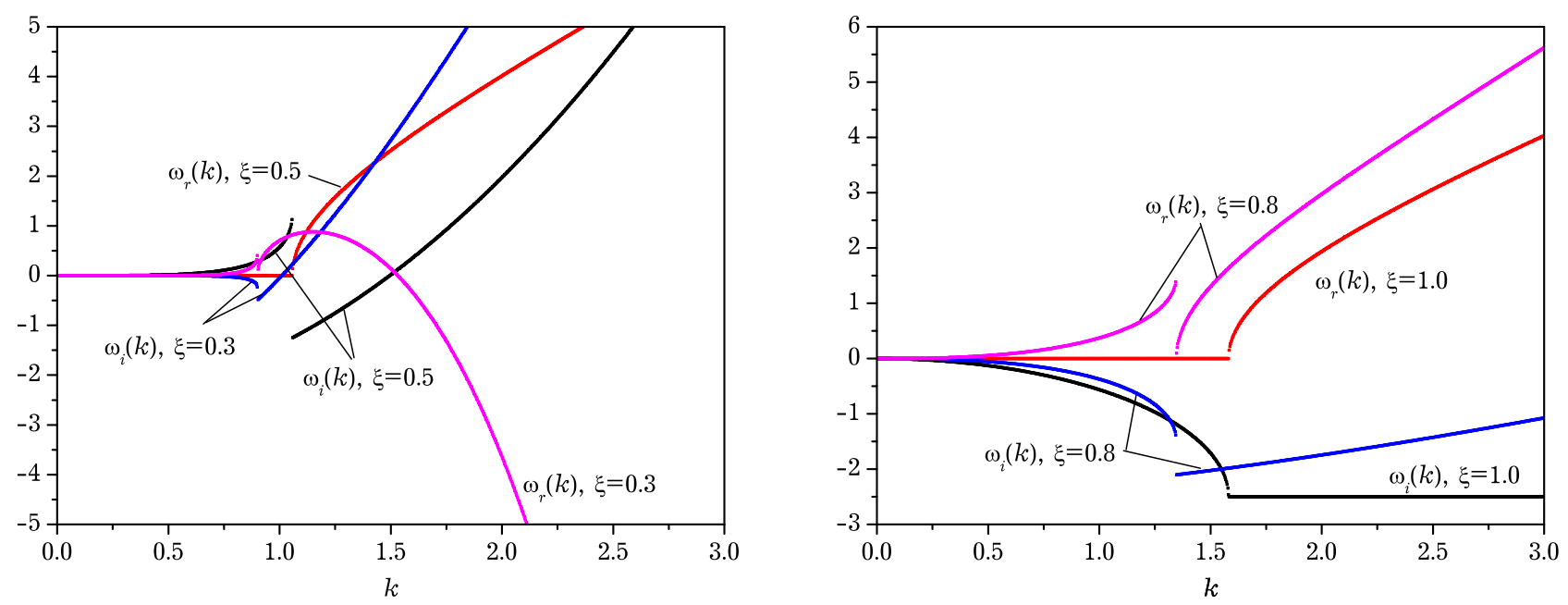

Fig. 1. The frequency spectrum for $\tau=0.2, \overline{D^{\prime}}=0.5$.
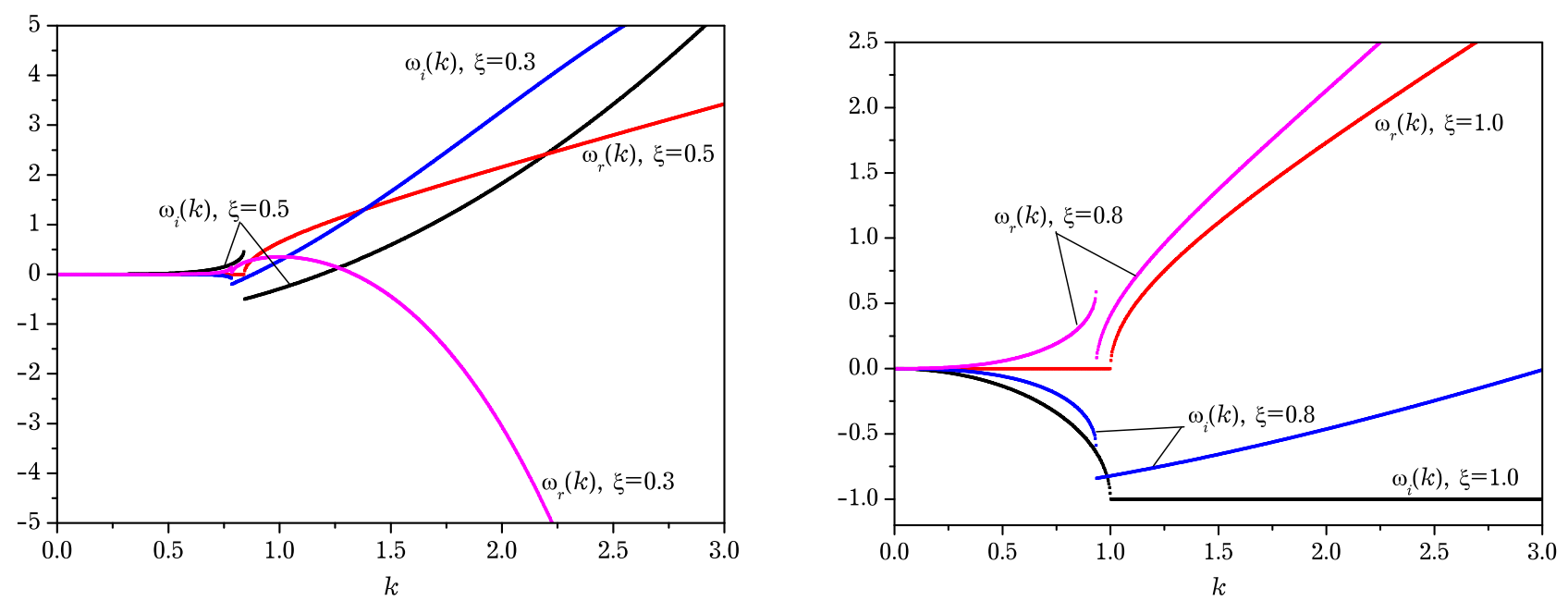

Fig. 2. The frequency spectrum for $\tau=0.5, \overline{D^{\prime}}=0.5$.
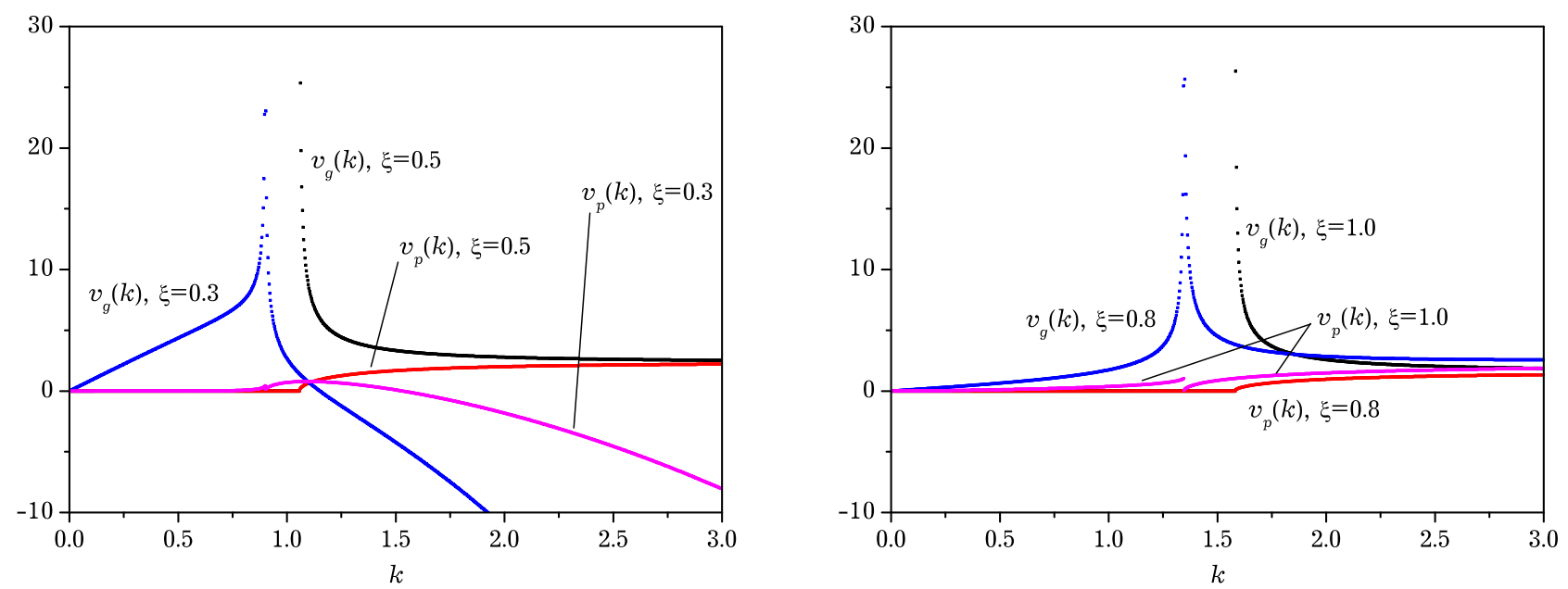

Fig. 3. The phase and group velocities for $\tau=0.2, \overline{D^{\prime}}=0.5$.

peak that, with increasing relaxation time, decreases. It is model research and it is obvious that features of the frequency spectrum studies would be important and interesting in investigating diffusion wave processes for specific real systems.

Mathematical Modeling and Computing, Vol.6, No. 1, pp. 58-68 (2019) 

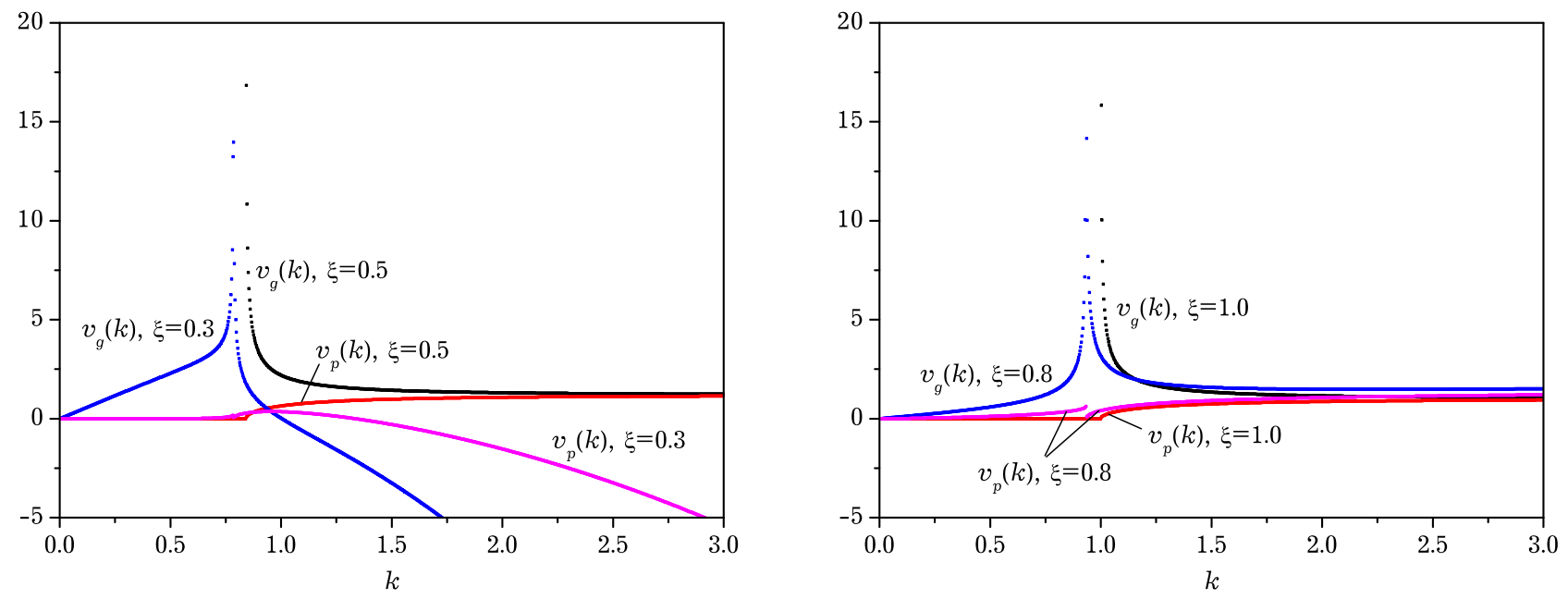

Fig. 4. The phase and group velocities for $\tau=0.5, \overline{D^{\prime}}=0.5$.

\section{Conclusions}

By using approach of Ref. [57,60], the new non-Markovian diffusion equations of particles in spatially heterogeneous environment with fractal structure have been obtained. By using approaches for the memory functions and fractional calculus [1-5], the generalized Cattaneo-Maxwell diffusion equations with taking into account nonlocality of space-time have been obtained. Dispersion relations for the Cattaneo-Maxwell-type diffusion equation are found, taking into account the time-spatial nonlocality in fractional derivatives. The frequency spectrum, phase and group velocities of the particles have been calculated for two values of the relaxation time $\tau=0.2,0.5$ and the diffusion coefficient $\overline{D^{\prime}}=0.5$ with the change in the value of the order of the fractional derivative $\xi=0.3,0.5,0.8,1$. It is shown that the frequency spectrum of the diffusion process is of wave behavior with discontinuities, which also manifests itself in the behavior of the phase velocity.

[1] Oldham K. B., Spanier J. The Fractional Calculus: Theory and Applications of Differentiation and Integration to Arbitrary Order. Dover Books on Mathematics, Dover Publications (2006).

[2] Samko S. G., Kilbas A. A., Marichev O. I. Fractional Integrals and Derivatives: Theory and Applications. Gordon and Breach Science Publishers (1993).

[3] Podlubny I., Kenneth V.T.E. Fractional Differential Equations: An Introduction to Fractional Derivatives, Fractional Differential Equations, to Methods of Their Solution and Some of Their Applications. Mathematics in Science and Engineering 198, Academic Press (1998).

[4] Mandelbrot B. B. The fractal geometry of nature. W. H. Freeman and Company (1982).

[5] Uchaikin V.V. Fractional Derivatives Method. Artishock-Press, Uljanovsk (2008), (in Russian).

[6] Sahimi M. Non-linear and non-local transport processes in heterogeneous media: from long-range correlated percolation to fracture and materials breakdown. Physics Reports. 306 (4-6), 213-395 (1998).

[7] Korošak D., Cvikl B., Kramer J., Jecl R., Prapotnik A. Fractional calculus applied to the analysis of spectral electrical conductivity of clay-water system. Journal of Contaminant Hydrology. 92 (1-2), 1-9 (2007).

[8] Metzler R., Klafter J. The random walk's guide to anomalous diffusion: a fractional dynamics approach. Physics Reports. 339 (1), 1-77 (2000).

[9] Hilfer R. Fractional Time Evolution, chapter II, pp.87-130. World Scientific, Singapore, New Jersey, London, Hong Kong (2000).

[10] Bisquert J., Garcia-Belmonte G., Fabregat-Santiago F., Ferriols N. S., Bogdanoff P., Pereira E. C. Doubling Exponent Models for the Analysis of Porous Film Electrodes by Impedance. Relaxation of $\mathrm{TiO}_{2}$ Nanoporous in Aqueous Solution. The Journal of Physical Chemistry. 104 (10), 2287-2298 (2000). 
[11] Bisquert J., Compte A. Theory of the electrochemical impedance of anomalous diffusion. Journal of Electroanalytical Chemistry. 499 (1), 112-120 (2001).

[12] Kosztołowicz T., Lewandowska K. D. Hyperbolic subdiffusive impedance. Journal of Physics A: Mathematical and Theoretical. 42 (5), 055004 (2009).

[13] Pyanylo Y.D., Prytula M. G., Prytula N. M., Lopuh N. B. Models of mass transfer in gas transmission systems. Mathematical Modeling and Computing. 1 (1), 84-96 (2014).

[14] Zhokh A., Trypolskyi A., Strizhak P. Relationship between the anomalous diffusion and the fractal dimension of the environment. Chemical Physics. 503, 71-76 (2018).

[15] Zhokh A. A., Strizhak P. E. Effect of zeolite ZSM-5 content on the methanol transport in the ZSM-5/alumina catalysts for methanol-to-olefin reaction. Chemical Engineering Research and Design. 127, 35-44 (2017).

[16] Zhokh A., Strizhak P. Non-Fickian diffusion of methanol in mesoporous media: Geometrical restrictions or adsorption-induced? The Journal of Chemical Physics. 146 (12), 124704 (2017).

[17] Scher H., MontrollE. W. Anomalous transit-time dispersion in amorphous solids. Phys. Rev. B. 12 (6), 2455-2477 (1975).

[18] Berkowitz B., Scher H. Theory of anomalous chemical transport in random fracture networks. Phys. Rev. E. 57 (5), 5858-5869 (1998).

[19] Bouchaud J. P., Georges A. Anomalous diffusion in disordered media: Statistical mechanisms, models and physical applications. Physics Reports. 195 (4), 127-293 (1990).

[20] Nigmatullin R. R. To the Theoretical Explanation of the "Universal Response". Physica Status Solidi (B). 123 (2), 739-745 (1984).

[21] Nigmatullin R. R. On the Theory of Relaxation for Systems with "Remnant" Memory. Physica Status Solidi (B). 124 (1), 389-393 (1984).

[22] Nigmatullin R. R. The realization of the generalized transfer equation in a medium with fractal geometry. Physica Status Solidi (B). 133 (1), 425-430 (1986).

[23] Nigmatullin R. R. Fractional integral and its physical interpretation. Theoretical and Mathematical Physics. 90 (3), 242-251 (1992).

[24] Nigmatullin R. R., Ryabov Y.E. Cole-Davidson dielectric relaxation as a self-similar relaxation process. Physics of the Solid State. 39 (1), 87-90 (1997).

[25] Nigmatullin R. R. Dielectric relaxation phenomenon based on the fractional kinetics: theory and its experimental confirmation. Physica Scripta. T136, 014001 (2009).

[26] Khamzin A. A., Nigmatullin R. R., Popov I. I. Microscopic model of a non-Debye dielectric relaxation: The Cole-Cole law and its generalization. Theoretical and Mathematical Physics. 173 (2), 1604-1619 (2012).

[27] Popov I. I., Nigmatullin R. R., Koroleva E. Y., Nabereznov A. A. The generalized Jonscher's relationship for conductivity and its confirmation for porous structures. Journal of Non-Crystalline Solids. 358 (1), 1-7 (2012).

[28] Grygorchak I. I., ～Kostrobij P. P., Stasjuk I. V., Tokarchuk M. V., Velychko O. V., Ivaschyshyn F. O., Markovych B. M. Fizichni procesy ta ih mikroskopichni modeli v periodychnyh neorganichno/organichnih klatratah. Rastr-7, Lviv (2015), (in Ukrainian).

[29] Kostrobij P. P., Grygorchak I. I., Ivaschyshyn F. O., Markovych B. M., Viznovych O. V., Tokarchuk M. V. Mathematical modeling of subdiffusion impedance in multilayer nanostructures. Mathematical Modeling and Computing. 2 (2), 154-159 (2015).

[30] Kostrobij P., Grygorchak I., Ivashchyshyn F., Markovych B., Viznovych O., Tokarchuk M. Generalized Electrodiffusion Equation with Fractality of Space-Time: Experiment and Theory. The Journal of Physical Chemistry A. 122 (16), 4099-4110 (2018).

[31] Balescu R. Anomalous transport in turbulent plasmas and continuous time random walks. Phys. Rev. E. 51 (5), 4807-4822 (1995).

[32] Tribeche M., Shukla P. K. Charging of a dust particle in a plasma with a non extensive electron distribution function. Physics of Plasmas. 18 (10), 103702 (2011).

[33] Gong J., Du J. Dust charging processes in the nonequilibrium dusty plasma with nonextensive power-law distribution. Physics of Plasmas. 19 (2), 023704 (2012). 
[34] Carreras B. A., Lynch V.E., Zaslavsky G. M. Anomalous diffusion and exit time distribution of particle tracers in plasma turbulence model. Physics of Plasmas. 8 (12), 5096-5103 (2001).

[35] Tarasov V. E. Electromagnetic field of fractal distribution of charged particles. Physics of Plasmas. 12 (8), 082106 (2005).

[36] Tarasov V.E. Magnetohydrodynamics of fractal media. Physics of Plasmas. 13 (5), 052107 (2006).

[37] Monin A. S. Uravnenija turbulentnoj difuzii. DAN SSSR, ser. geofiz. 2, 256-259 (1955), (in Russian).

[38] Klimontovich J. L. Vvedenie v fiziku otkrytyh sistem. Moskva, Janus (2002), (in Russian).

[39] Zaslavsky G. M. Chaos, fractional kinetics, and anomalous transport. Physics Reports. 371 (6), 461-580 (2002).

[40] Tarasov V. E. Fractional Dynamics: Applications of Fractional Calculus to Dynamics of Particles, Fields and Media. Nonlinear Physical Science, Springer Berlin Heidelberg (2010).

[41] Zaslavsky G. M. Fractional kinetic equation for Hamiltonian chaos. Physica D: Nonlinear Phenomena. 76 (1), 110-122 (1994).

[42] Saichev A. I., Zaslavsky G. M. Fractional kinetic equations: solutions and applications. Chaos. 7 (4), $753-$ 764 (1997).

[43] Zaslavsky G. M., Edelman M. A. Fractional kinetics: from pseudochaotic dynamics to Maxwell's Demon. Physica D: Nonlinear Phenomena. 193 (1-4), 128-147 (2004).

[44] Nigmatullin R. 'Fractional' kinetic equations and 'universal' decoupling of a memory function in mesoscale region. Physica A: Statistical Mechanics and its Applications. 363 (2), 282-298 (2006).

[45] Chechkin A. V., Gonchar V.Y., Szydłowski M. Fractional kinetics for relaxation and superdiffusion in a magnetic field. Physics of Plasmas. 9 (1), 78-88 (2002).

[46] Gafiychuk V.V., Datsko B.Y. Stability analysis and oscillatory structures in time-fractional reactiondiffusion systems. Phys. Rev. E. 75 (5), 055201 (2007).

[47] Kosztołowicz T., Lewandowska K. D. Time evolution of the reaction front in a subdiffusive system. Phys. Rev. E. 78 (6), 066103 (2008).

[48] Shkilev V. P. Subdiffusion of mixed origin with chemical reactions. Journal of Experimental and Theoretical Physics. 117 (6), 1066-1070 (2013).

[49] Hobbie R. K., Roth B. J. Intermediate Physics for Medicine and Biology. Springer-Verlag, New York (2007).

[50] Jeon J. H., Monne H. M. S., Javanainen M., Metzler R. Anomalous Diffusion of Phospholipids and Cholesterols in a Lipid Bilayer and its Origins. Phys. Rev. Lett. 109 (18), 188103 (2012).

[51] Höfling F., Franosch T. Anomalous transport in the crowded world of biological cells. Reports on Progress in Physics. 76 (4), 046602 (2013).

[52] Uchaikin V.V. Fractional phenomenology of cosmic ray anomalous diffusion. Physics-Uspekhi. 56 (11), 1074-1119 (2013).

[53] Szymanski J., Weiss M. Elucidating the Origin of Anomalous Diffusion in Crowded Fluids. Phys. Rev. Lett. 103 (3), 038102 (2009).

[54] Sandev T., Tomovski Z., Dubbeldam J. L. A., Chechkin A. Generalized diffusion-wave equation with memory kernel. Journal of Physics A: Mathematical and Theoretical. 52 (1), 015201 (2018).

[55] Sandev T., Metzler R., Chechkin A. Generalised Diffusion and Wave Equations: Recent Advances. arXiv:1903.01166 (2019).

[56] Giusti A. Dispersion relations for the time-fractional Cattaneo-Maxwell heat equation. Journal of Mathematical Physics. 59 (1), 013506 (2018).

[57] Kostrobij P., Markovych B., Viznovych O., Tokarchuk M. Generalized diffusion equation with fractional derivatives within Renyi statistics. Journal of Mathematical Physics. 57 (9), 093301 (2016).

[58] Kostrobij P., Markovych B., Viznovych O., Tokarchuk M. Generalized electrodiffusion equation with fractality of space-time. Mathematical Modeling and Computing. 3 (2), 163-172 (2016).

[59] Glushak P. A., Markiv B. B., Tokarchuk M. V. Zubarev's Nonequilibrium Statistical Operator Method in the Generalized Statistics of Multiparticle Systems. Theoretical and Mathematical Physics. 194 (1), 57-73 (2018). 
[60] Kostrobij P., Markovych B., Viznovych O., Tokarchuk M. Generalized transport equation with nonlocality of space-time. Zubarev's NSO method. Physica A: Statistical Mechanics and its Applications. 514, 63-70 (2019).

[61] Zubarev D. N. Modern methods of the statistical theory of nonequilibrium processes. Journal of Soviet Mathematics. 16 (6), 1509-1571 (1981).

[62] Zubarev D. N., Morozov V. G., Röpke G. Statistical mechanics of nonequilibrium processes. Vol. 1. Moscow, Fizmatlit (2002), (in Russian).

[63] Zubarev D. N., Morozov V. G., Röpke G. Statistical mechanics of nonequilibrium processes. Vol. 2. Moscow, Fizmatlit (2002), (in Russian).

[64] Markiv B., Tokarchuk R., Kostrobij P., Tokarchuk M. Nonequilibrium statistical operator method in Renyi statistics. Physica A: Statistical Mechanics and its Applications. 390 (5), 785-791 (2011).

[65] Cottrill-Shepherd K., Naber M. Fractional differential forms. Journal of Mathematical Physics. 42 (5), 2203-2212 (2001).

[66] Mainardi F. Fractional Calculus. Springer, Vienna (1997).

[67] Caputo M., MainardiF. A new dissipation model based on memory mechanism. Pure and Applied Geophysics. 91 (1), 134-147 (1971).

\title{
Узагальнене рівняння дифузії Кеттано-Максвелла у дробових похідних. Дисперсійні співвідношення
}

\author{
Костробій П. ${ }^{1}$, Маркович Б. ${ }^{1}$, Візнович O. ${ }^{1}$, Зелінська I. ${ }^{1}$, Токарчук M. ${ }^{1,2}$ \\ ${ }^{1}$ Національний університет "Лъвівська політехніка", \\ вул. С. Бандери, 12, Львів, 79013, Україна \\ ${ }^{2}$ Інститут фізики конденсованих систем НАН Украйни, \\ вул. Свениіиьвкого, 1, Львів, 79011, Україна
}

Отримано нове немарковське рівняння дифузії частинок у просторово неоднорідному середовищі з фрактальною структурою та узагальнене рівняння дифузії Кеттано-Максвелла з урахуванням просторово-часової нелокальності. Знайдено дисперсійні співвідношення для рівняння дифузії типу Кеттано-Максвелла з урахуванням просторово-часової нелокальності у дробових похідних. Розраховано частотний спектр, фазову та групову швидкості й показано його хвильову поведінку зі стрибкоподібними розривами, які проявляються також у зміні фазової швидкості.

Ключові слова: узагальнене рівняння дифузї̈, нерівноважний статистичний оператор, статистика Рені, часова мультифрактальність, просторова фрактальність, просторово-часова фрактальність.

2000 MSC: $82 \mathrm{C} 05,82 \mathrm{C} 70$

Удк: 538.93 